\title{
Time-driven Activity-based Costing More Accurately Reflects Costs in Arthroplasty Surgery
}

\author{
Sina Akhavan BA, Lorrayne Ward MBA, MPP, \\ Kevin J. Bozic MD, MBA
}

Published online: 27 February 2015

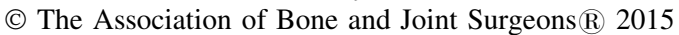

\begin{abstract}
Background Cost estimates derived from traditional hospital cost accounting systems have inherent limitations that restrict their usefulness for measuring process and quality improvement. Newer approaches such as time-driven activity-based costing (TDABC) may offer more precise estimates of true cost, but to our knowledge, the
\end{abstract}

The analyses on which this publication is based were performed under a grant funded by the California Healthcare Foundation.

All ICMJE Conflict of Interest Forms for authors and Clinical

Orthopaedics and Related Research ${ }^{\circledR}$ editors and board members are on file with the publication and can be viewed on request.

Clinical Orthopaedics and Related Research ${ }^{\mathbb{R}}$ neither advocates nor endorses the use of any treatment, drug, or device. Readers are encouraged to always seek additional information, including FDAapproval status, of any drug or device prior to clinical use.

Each author certifies that his or her institution approved the human protocol for this investigation, that all investigations were conducted in conformity with ethical principles of research, and that informed consent for participation in the study was obtained.

This work was performed at the University of California, San Francisco, CA, USA.

\section{S. Akhavan}

University of California, San Francisco, School of Medicine,

San Francisco, CA, USA

L. Ward

Performance Excellence Department, University of California San Francisco Medical Center, San Francisco, CA, USA

\section{K. J. Bozic ( $\square)$}

Department of Orthopaedic Surgery, University of California, San Francisco, 500 Parnassus Avenue MUW317, San Francisco, CA 94143-0728, USA

e-mail: bozick@orthosurg.ucsf.edu; kevin.bozic@ucsf.edu

\section{K. J. Bozic}

Philip R. Lee Institute for Health Policy Studies, University of California, San Francisco, San Francisco, CA, USA differences between this TDABC and more traditional approaches have not been explored systematically in arthroplasty surgery.

Questions/purposes The purposes of this study were to compare the costs associated with (1) primary total hip arthroplasty (THA); (2) primary total knee arthroplasty (TKA); and (3) three surgeons performing these total joint arthroplasties (TJAs) as measured using TDABC versus traditional hospital accounting (TA).

Methods Process maps were developed for each phase of care (preoperative, intraoperative, and postoperative) for patients undergoing primary TJA performed by one of three surgeons at a tertiary care medical center. Personnel costs for each phase of care were measured using TDABC based on fully loaded labor rates, including physician compensation. Costs associated with consumables (including implants) were calculated based on direct purchase price. Total costs for 677 primary TJAs were aggregated over 17 months (January 2012 to May 2013) and organized into cost categories (room and board, implant, operating room services, drugs, supplies, other services). Costs derived using TDABC, based on actual time and intensity of resources used, were compared with costs derived using TA techniques based on activity-based costing and indirect costs calculated as a percentage of direct costs from the hospital decision support system.

Results Substantial differences between cost estimates using TDABC and TA were found for primary THA (USD 12,982 TDABC versus USD 23,915 TA), primary TKA (USD 13,661 TDABC versus USD 24,796 TA), and individually across all three surgeons for both (THA: TDABC $=49 \%-55 \%$ of TA total cost; TKA: TDABC $=$ $53 \%-55 \%$ of TA total cost). Cost categories with the most variability between TA and TDABC estimates were operating room services and room and board. 
Conclusions Traditional hospital cost accounting systems overestimate the costs associated with many surgical procedures, including primary TJA. TDABC provides a more accurate measure of true resource use associated with TJAs and can be used to identify high-cost/high-variability processes that can be targeted for process/quality improvement.

Level of Evidence Level III, therapeutic study.

\section{Introduction}

The American healthcare system is facing a period of unprecedented change. National healthcare expenditures continue to outpace inflation, nearing $18 \%$ of the gross domestic product (GDP), and are expected to increase to $20 \%$ of GDP by 2020 [4]. Healthcare stakeholders must work collaboratively to improve quality, lower costs, and thus foster better value (as defined by health outcomes achieved per dollar spent) in medicine [7].

To improve value in health care, providers must have a better understanding of the costs and outcomes associated with specific healthcare interventions open to change. If costs and outcomes are not measured in sufficient detail, it is impossible to evaluate the impact of changes in systems and processes of care [2]. Healthcare provider organizations generally use one of the following costing methods or a hybrid method to estimate the costs associated with the services they deliver: ratio of cost to charges, activity-based costing, or time-driven activitybased costing.

\section{Ratio of Cost to Charges}

Cost information is estimated within departments by multiplying revenues generated by the ratio of treatment costs to charges set by the hospital chargemaster, which includes hospital services, procedures, equipment fees, drugs, supplies, etc. Each item is assigned a unique identifier code and a set price used to generate patient bills. Every hospital system maintains its own chargemaster. There are three key assumptions in ratio of cost to charges methodology: (1) indirect costs are from a single pool (eg, that allocated costs are interchangeable); (2) reimbursement rates reflect intensity of services provided; and (3) each type of service consumes indirect costs in the same proportion [8]. In the ratio of cost to charges methodology, the emphasis is placed on maximizing revenue as opposed to controlling costs because of a bias toward profitability of activities.
Activity-based Costing

Activity-based costing (ABC) is a costing methodology that identifies activities necessary to produce a defined service or product and assigns the cost of each activity resource to processes according to the actual consumption associated with the provision of activity. Cost assignment through ABC occurs in two stages: processes consume activities and activities consume costs. Indirect costs are thus allocated to processes on the basis of the activities consumed within the provision of those services. The ABC method is therefore inherently more accurate than the ratio of cost to charges and can be used to identify inefficient services and reengineer care to optimize value. Implementing $\mathrm{ABC}$ is both time- and resource-intensive. Many healthcare managers who have tried to implement $\mathrm{ABC}$ in their organizations have abandoned the attempt in the face of rising costs and employee pushback as a result of the burden of frequent interviews, time logs, and direct observations [5].

\section{Time-driven Activity-based Costing}

Kaplan and Anderson developed a variant of $\mathrm{ABC}$, called time-driven ABC (TDABC) [2]. TDABC models can be implemented relatively quickly because only estimates of two parameters are required: (1) the unit cost of supplying capacity (eg, cost per minute of a surgeon's time); and (2) the time required to perform a transaction or an activity. The breakthrough of TDABC lies in using time equations to estimate the time spent on each activity [3]. Through the use of multiple time drivers, TDABC can capture operational processes with detail easily and quickly, is less expensive to update, and can provide opportunities to design cost models more simply than ABC [2].

The purpose of this study was to measure the costs associated with (1) primary THA; (2) primary TKA; and (3) three different surgeons performing those procedures at our institution using TDABC and to compare those cost estimates with those derived using traditional hospital accounting (TA) methods.

\section{Materials and Methods}

\section{Time-driven Activity-based Costing}

The implementation of TDABC requires three separate steps: (1) creation of process maps; (2) estimation of costs associated with the provision of specific services; and (3) 


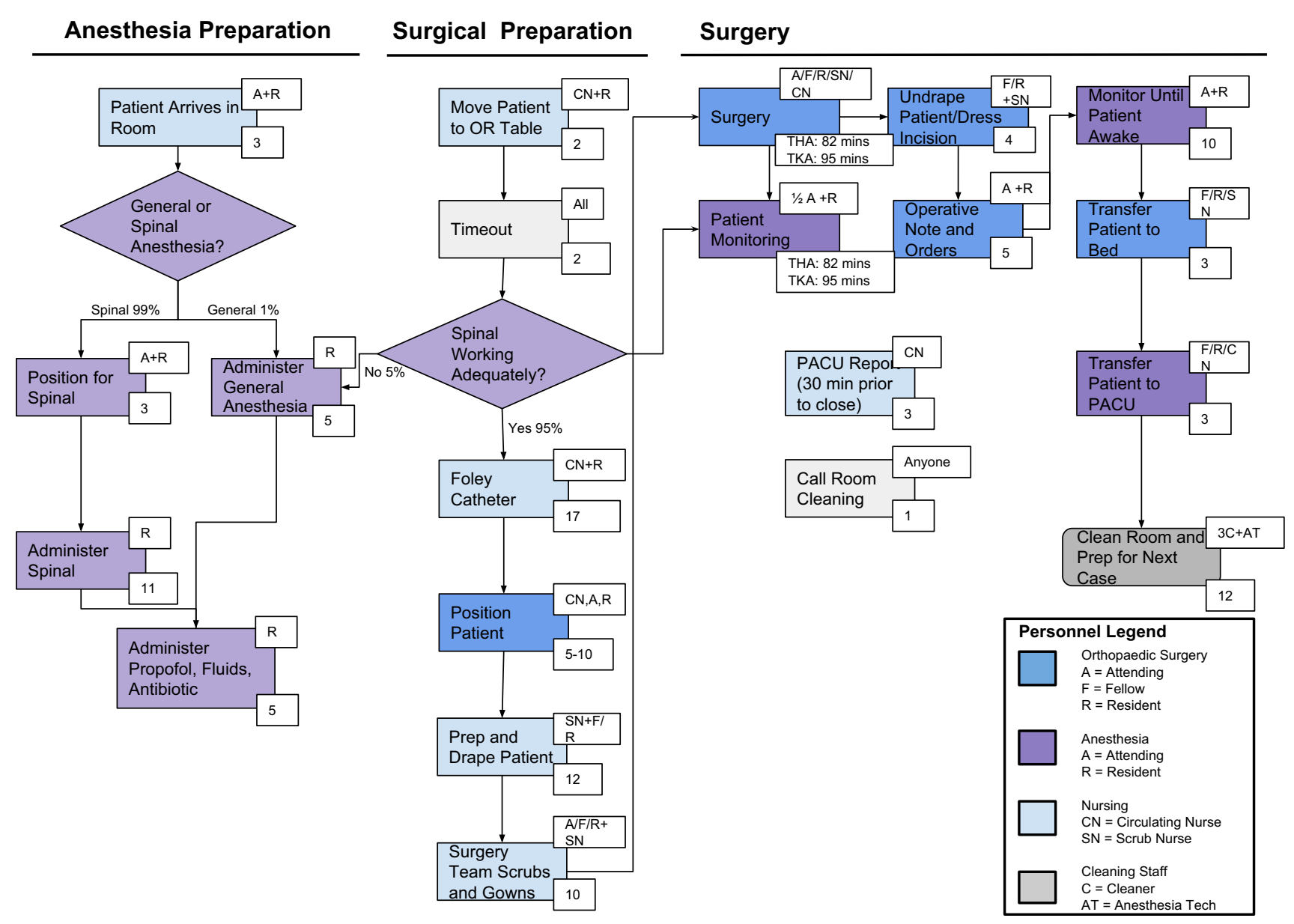

Fig. 1 Diagram shows a process map outline of the arthroplasty operating day including anesthesia preparation, surgical preparation, and surgery. Large boxes represent activities with arrows indicating sequence. Colors correspond to personnel categories with personnel ID

aggregation of total costs through synthesis of cost and time data.

\section{Process Maps}

Process maps were generated for primary unilateral THAs/ TKAs from the initial clinic visit to hospital discharge for three orthopaedic surgeons to understand how patients progress through the care cycle, quantify human resources use by activity, and identify areas of variation and inefficiency. Process maps, along with time estimates for each step, were created by 8 weeks of direct observation, selfreporting of unobserved documentation time, and multidisciplinary validation of care maps through meetings with frontline staff and management.

All resources (capacity resources such as personnel and consumable supplies such as implants) required for each process step were identified (Fig. 1). in the upper smaller boxes (see legend). Numbers in smaller boxes correspond to minutes used per activity. Process maps provide individual personnel time estimates that are used to obtain cost data per activity. $\mathrm{OR}=$ operating room; PACU $=$ postanesthesia care unit.

Estimation of Costs Associated with Provision of Specific Services

To measure costs, we estimated the cost of supplying each service based on the time required for each resource type as well as the practical capacity (defined subsequently) of each resource (eg, a surgeon or nurse practitioner), and then we computed total costs over each patient's cycle of care. First, we identified the groups of resources (eg, surgeons, nurses, etc) that perform activities for patients seen at our institution for THA and TKA. Costs were organized into categories: implant cost, room and board, drug cost, supplies (eg, operating room consumables), and other services (eg, respiratory therapy). Fully loaded labor rates (eg, salary plus benefits) for nonphysicians were calculated based on 2012 expenses and average full-time equivalents (FTEs). A total of 3244 medical center general ledger accounts with over USD 641 million in total expense dollars were used in the analysis. Physician compensation rates 
were calculated outside of the model based on yearly salary, fringe benefits, and time dedicated to clinical duties (eg, not research, teaching, and other administrative duties).

Transaction data was comprised of over 17 months of patient information (January 2012 through May 2013) with 677 patients receiving 700 primary total joint arthroplasties (TJAs). All patients with a qualifying procedure and inpatient stay during the period were included in the analysis. Direct costs were input for implants, operating room, supplies, and drugs. Individual processes were defined in the model from inpatient process maps with each process representing an activity performed for a specific job code. Resource rates for the process maps were calculated using general ledger-based rate calculations. For example, general ledger-based rate calculations: 2012 fully loaded cost per FTE type/(2012 number of FTEs $\times$ FTE capacity/year). Fully loaded costs were calculated using employee wage rates, number of hours available (the practical capacity), and additional costs such as benefits, insurance, training, etc. Theoretical capacity is the expected available working minutes for a full-time FTE. The practical capacity was estimated to be $80 \%$ of the theoretical capacity to account for nonused time (ie, breaks, vacation, conversations, and other activity unrelated to patient care) [2]. By dividing the yearly cost per resource by the practical capacity, the perminute cost of each resource is calculated. Indirect costs under TDABC were assigned based on how they are "consumed" by the activities in question, as opposed to being tacked on at a standard rate regardless of use. For example, for costs related to space such as facilities maintenance and utilities, the overall expenditures associated with those areas is assigned as a ratio of the square footage used by the activities out of the total square footage of the institution. Therefore, under TDABC, activities using a great deal of space would be charged a higher amount for space-related indirect costs, regardless of the direct cost of those activities. Indirect costs related to FTEs such as human resources support, finance, and information technology support are allocated based on the number of FTEs associated with the activities in question. Applying the perminute cost to different points of the process map allowed for calculation of the activity cost for each activity during the patient care cycle. With the exception of operating room events, all activity times were based on process maps. Activity times were collected from the process maps and only "used capacity" costs were allocated to the transactions.

\section{Aggregation of Costs}

Each step in the process map was associated with a specific resource and time necessary for activity completion based on the most commonly observed combination of resource type and time. For each activity there was an associated personnel resource and resource rate. The resource rates were calculated using methods in Step 2. To calculate cost, time necessary for each activity/step is multiplied by resources used for that particular activity/step; total cost is thus the summation of costs of all activities for each care cycle. The per-patient process cost for care delivered was estimated for each step in the process maps based on time estimates and resource rates.

\section{Traditional Accounting}

Traditional accounting in our institution uses an ABCbased methodology through the hospital decision support system (EPSi; Allscripts, Inc, Libertyville, IL, USA) that allocates expenses from the general ledger and payroll to activity codes. Codes are derived from billable items and operational events captured in the chargemaster. All costs of providing services are grouped by cost types and categories (which include indirect costs) and allocated costs across units of service. Units of service are aggregated to determine the cost of a patient's care cycle. Triggers for costs for supplies and implants are derived from the general ledger; other costs such as inpatient stay days are based on a fixed rate per day by a specific nursing unit (for example postsurgical unit or floor unit). Indirect costs that include items such as management, information technology, finance, and facilities require assumptions for cost allocation and are calculated as $60 \%$ of the direct costs. For example, an implant direct cost of USD 6000 is assigned an indirect cost of USD 3600 totaling USD 9600. Thus, total costs equal direct costs in addition to $60 \%$ of the direct costs.

Inpatient costs from Fiscal Year 2012 were collected and grouped into the following cost categories: implant cost, room and board, drug cost, supplies, and other services. Cost data were gathered retroactively only for Fiscal Year 2012 because it very closely mirrored Fiscal Year 2013 cost data.

\section{Results}

Primary THA TDABC versus TA

Costs derived using TDABC were estimated at $54 \%$ of costs derived using TA for primary THA (USD 12,982 TDABC versus USD 23,915 TA), accounting for a USD 10,933 difference. Cost differences were found in every cost category with the following TDABC to TA ratios (TDABC/TA): implant cost (62\%) room and board (55\%), operating room services (39\%), drug cost (42\%) and supplies (60\%), and other services (101\%) (Fig. 2). 


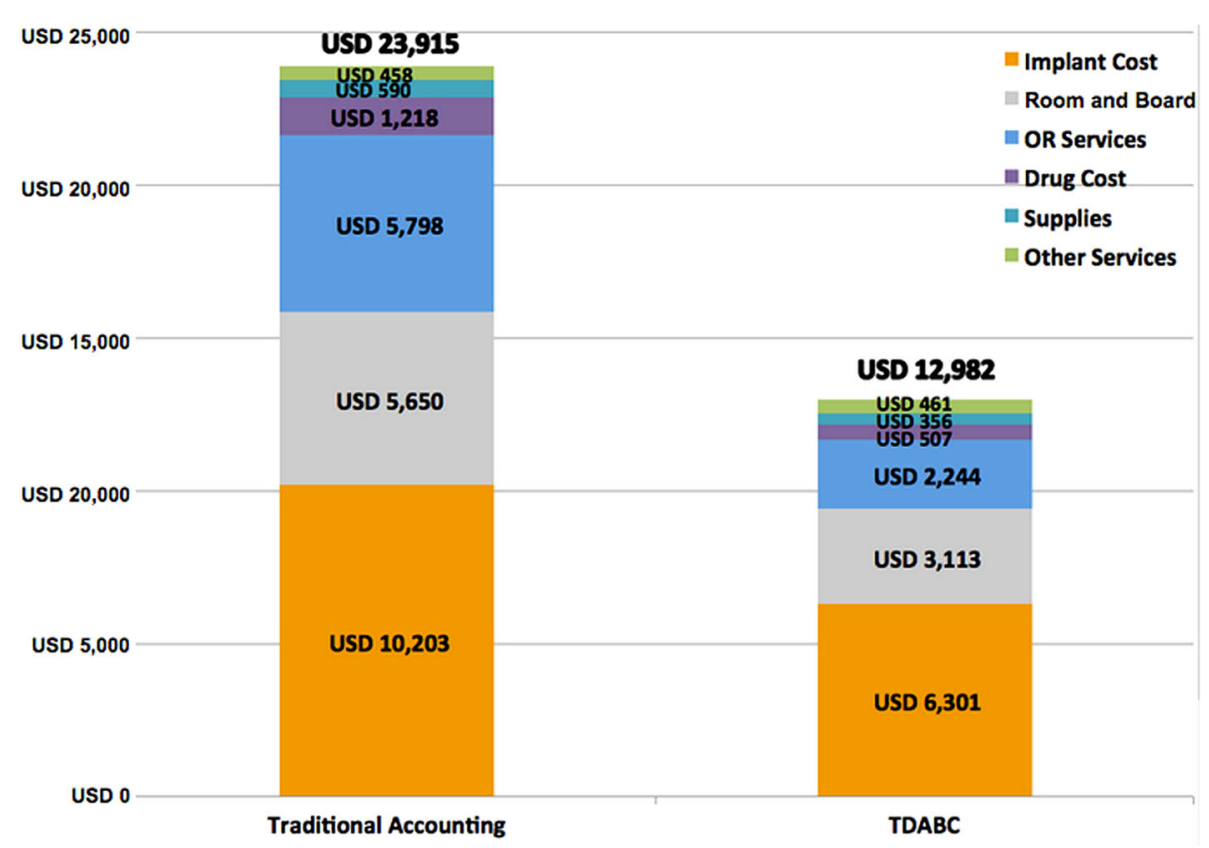

Fig. 2 Graph shows TDABC versus TA in THA. TDABC analysis reveals lower costs in every major cost category. OR $=$ operating room.

Primary TKA TDABC versus TA

Costs derived using TDABC were $55 \%$ of costs derived using TA for primary TKA (USD 13,661 TDABC versus USD 24,796 TA) accounting for a USD 11,135 difference. Cost differences were found in every cost category with the following TDABC to TA ratios (TDABC/TA): implant cost $(66 \%)$, room and board (54\%), operating room services $(38 \%)$, drug cost $(52 \%)$ and supplies $(51 \%)$, and other services (114\%) (Fig. 3).

Surgeons Performing Total Joint Arthroplasty TDABC versus TA

\section{Comparing Individual Surgeons for Primary THA}

Surgeon A's TDABC costs were $55 \%$ of TA costs in primary THA (USD 12,172 TDABC versus USD 22,093 TA) accounting for a USD 9321 difference. Cost differences were found in every cost category with the following TDABC to TA ratios (TDABC/TA): implant cost $(61 \%)$, room and board (57\%), operating room services (39\%), drug cost (55\%), supplies (58\%), and other services (99\%).

Surgeon B's TDABC costs were $49 \%$ of TA costs in primary THA (USD 14,883 TDABC versus USD 30,207 TA) accounting for a USD 15,324 difference. Cost differences were found in every cost category with the following TDABC to TA ratios (TDABC/TA): implant cost (61\%), room and board (52\%), operating room services (36\%) drug cost (24\%), supplies (59\%), and other services (143\%).
Surgeon C's TDABC costs were $55 \%$ of TA costs in primary THA (USD 13,277 TDABC versus USD 24,265 TA) accounting for a USD 10,988 difference. Cost differences were found in every cost category with the following TDABC to TA ratios (TDABC/TA): implant cost $(61 \%)$, room and board (53\%), operating room services (39\%), drug cost (74\%), supplies (66\%), and other services (92\%) (Fig. 4).

\section{Comparing Individual Surgeons for Primary TKA}

Surgeon A's TDABC costs were $55 \%$ of TA costs in primary TKA (USD 12,095 TDABC versus USD 21,977 TA) accounting for a USD 9882 difference. Cost differences were found in every cost category with the following TDABC to TA ratios (TDABC/TA): implant cost $(65 \%)$, room and board $(54 \%)$, operating room services $(39 \%)$, drug cost (64\%), supplies (55\%), and other services (106\%).

Surgeon B's TDABC costs were $53 \%$ of TA costs in primary TKA (USD 17,377 TDABC versus USD 32,922 TA) accounting for a USD 15,545 difference. Cost differences were found in every cost category with the following TDABC to TA ratios (TDABC/TA): implant cost (65\%), room and board (52\%), operating room services (38\%), drug cost (36\%), supplies (40\%), and other services (141\%).

Surgeon C's TDABC costs were 53\% of TA costs in primary TKA (USD 13,169 TDABC versus USD 24,952 TA) accounting for a USD 11,783 difference. Cost differences were found for every cost category with the following TDABC to TA ratios (TDABC/TA): implant cost $(61 \%)$, room and board (51\%), operating room 


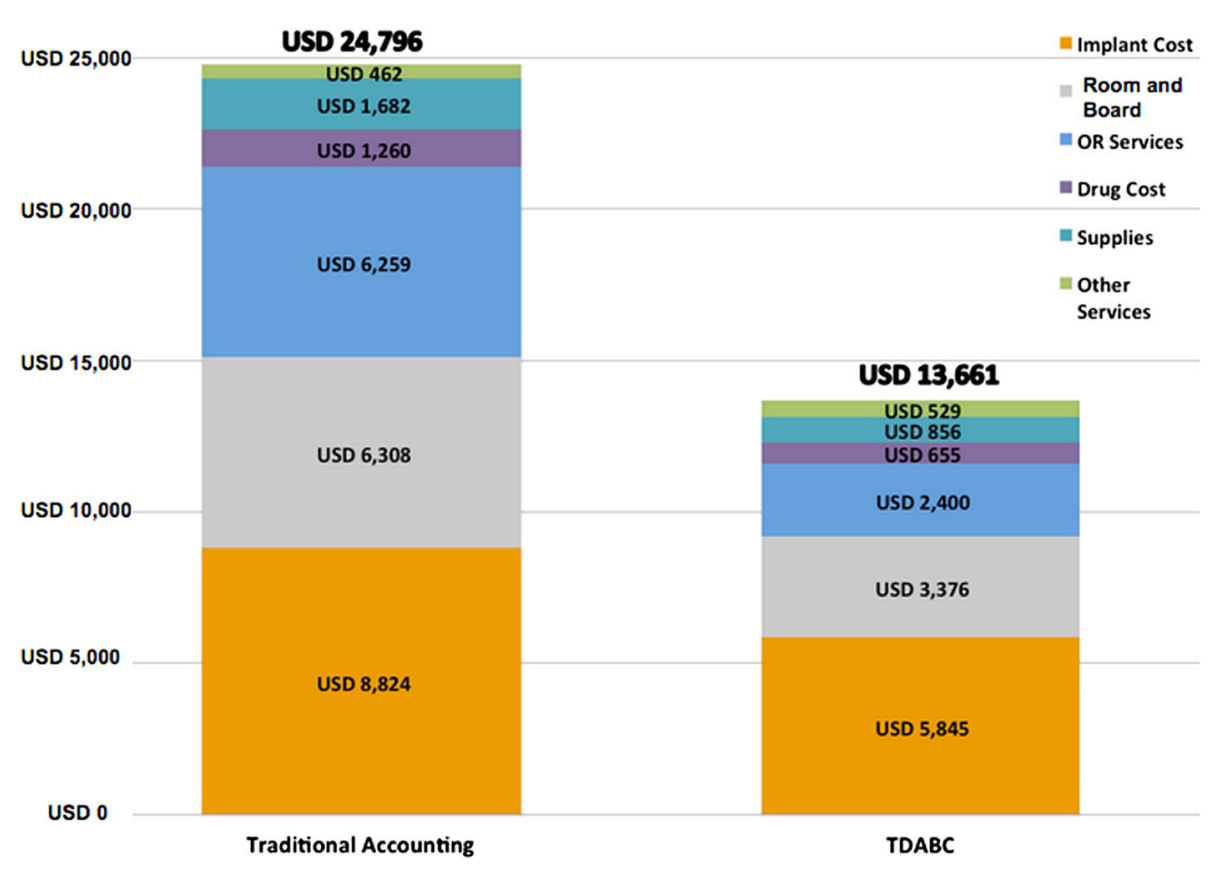

Fig. 3 Graph shows TDABC versus TA in TKA. TDABC analysis reveals lower costs in every major cost category. OR = operating room.

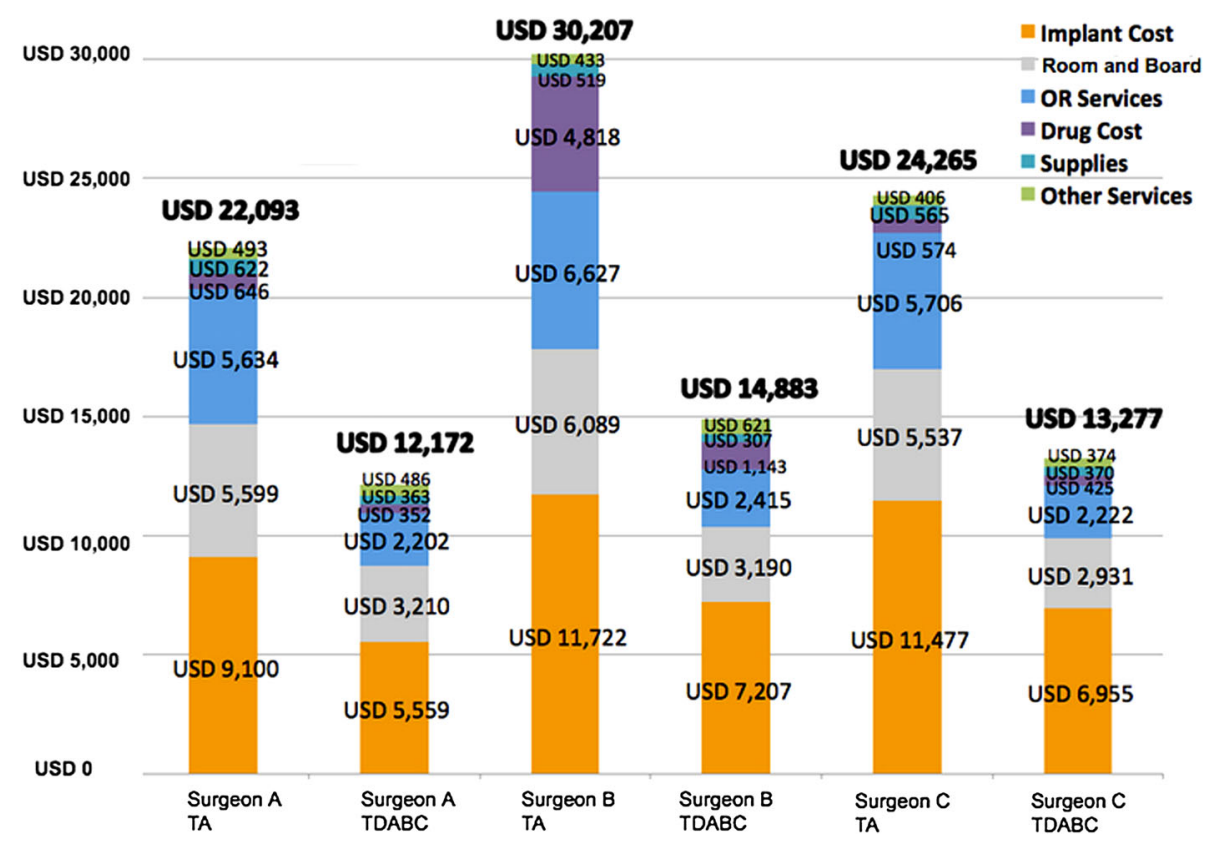

Fig. 4 Graph shows TDABC versus TA in THA among three surgeons. TDABC analysis reveals lower costs in every major cost category. Primary driver for differences is in implant costs. $\mathrm{OR}=$ operating room.

services (37\%), drug cost (64\%) and supplies (54\%), and other services (107\%) (Fig. 5).

\section{Discussion}

As hospitals move toward value-based payments (such as bundled payments), there is increased need to understand true costs for specific service lines. TDABC has been developed to obtain better granularity on utilized costs. The Cleveland Clinic recently used TDABC and found it to produce a cost $10 \%$ lower than conventional accounting in cardiac surgery [1]. TDABC has also been used to improve understanding of actual costs and care redesign engagement in neurosurgery and urology [6]. Our goal was to observe if TDABC could provide more accurate costing 


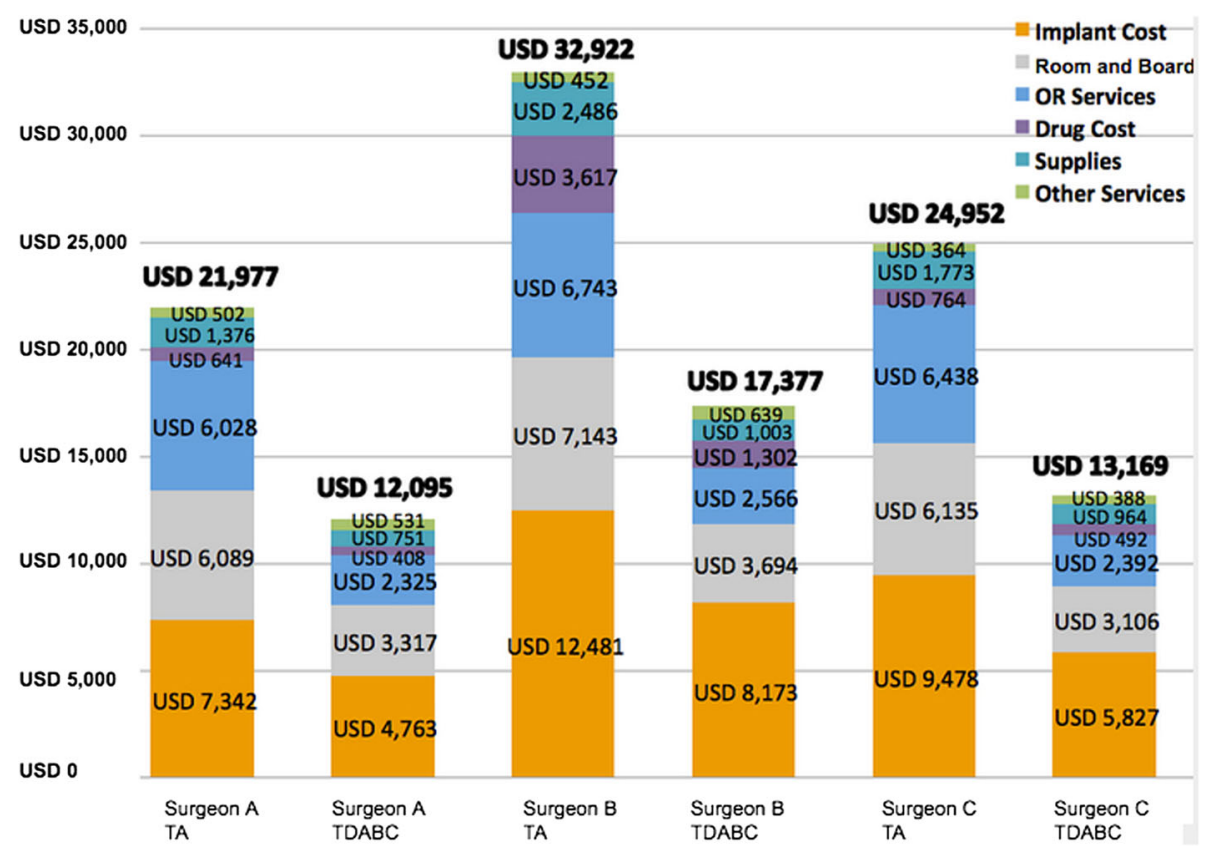

Fig. 5 Graph shows TDABC versus TA in TKA among three surgeons. TDABC analysis reveals lower costs in every major cost category. Primary driver for differences is in implant costs. $\mathrm{OR}=$ operating room.

data than traditional accounting for TJA. Our primary goal was to compare costs derived from TDABC and TA in primary THA and TKA at our institution. This study demonstrates how TDABC can be used to measure actual resource use associated with primary TJA. Primary THA and TKA were shown to cost less (USD 10,933 and USD 11,833 , respectively) using TDABC compared with traditional hospital accounting methods. Furthermore, cost differences among individual surgeons for THA (Surgeon A: USD 9921; Surgeon B: USD 15,324; Surgeon C: USD 10,988) and TKA (Surgeon A: USD 9882; Surgeon B: USD 15,545; Surgeon C: USD11,783) were found between $\mathrm{TA}$ and TDABC.

There are inherent challenges in comparing TDABC with TA, however. The differences in total costs are in large part attributable to indirect cost allocation methodology used in TA. Thus, the lower costs found in TDABC do not suggest that current TA cost values are incorrect. TDABC and TA are simply two different accounting methods. TDABC treats indirect costs as a resource consumed or "used capacity," whereas TA in our institution approximates indirect costs as $60 \%$ of the direct costs with the assumption that services with greater direct cost consume more indirect cost as well. Furthermore, our TDABC process was not able to fully account for all indirect and related costs to TJA (such as research or sterile processing), but did account for facilities, equipment, information technology, and most other traditional "overhead" costs. Time spent on patient care outside of the patient interaction is difficult to account for such as in coordination of care or interprovider communication. Additionally, process maps are resource-intensive to create and are only time estimates based on employee and supervisor estimations (if not directly observed). The application of process maps across a hospital system would be difficult to achieve, although maps can be carefully applied to high-volume, low-variability service lines to identify areas of improvement. In addition, many newer electronic health record systems have detailed time stamps for key care processes, which could facilitate a more accurate TDABC effort in real time.

Primary THA and TKA using TDABC were found to be at $54 \%$ and $55 \%$ of TA costs. The majority of the differences in cost in each category originate from indirect costs lumped into all direct costs as a percentage in addition to unused capacity being captured by TA.

Differences among surgeons are accounted for similarly: TA costs are high as a result of unused capacity and indirect costs. Differences in TJA costs between surgeons were related to implant preferences and corresponding acquisition costs.

Using process maps and TDABC, one can obtain granular analysis of cost and time that can be used to inform process improvement initiatives. By applying TDABC in this study, we were able to illustrate differences compared with our TA methods. Notably, variability in cost estimates was found in every cost category for THA and TKA. Implants, supplies, and drug costs are consumables whose 
acquisition costs are independent of costing methodology and differences are reflections of the indirect costs added in when using traditional hospital cost accounting. TDABC accounts for only the time where the equipment or personnel is used in delivering a specific service, whereas TA allocates all equipment and personnel expenses to the activity. Differences in operating room services and room and board reflect the ability of TDABC to allocate direct and indirect costs at a more detailed level by the used capacity for a particular service. TA allocates all expenses, including unused capacity, to each unit of service.

Using TDABC, clinicians and administrators can view transparent cost analyses and redesign processes to reduce cost and deliver care with similar (or improved) outcomes. Clinical use can be reviewed and used to develop standardized protocols across providers. As value-based payment models such as bundled payments, which require a detailed understanding of costs, are becoming more accepted, TDABC provides a valuable method of measuring costs associated with an episode of care. In episode-based payments, payers negotiate with providers to establish a set amount to reimburse for each episode of care; providers are responsible for costs beyond the set amount. The provider must understand costs to negotiate a sustainable bundled payment that will adequately cover their costs. Securing competitive bundle payment contracts can lead to increased volume for a provider network. A bottom-up approach with cost and process analysis through TDABC allows clarity of resources used and ability to redesign care cycles to deliver better value to patients.

Acknowledgments We thank Matt Callahan for helping attaining TA costs for our study.

\section{References}

1. Donovan CJ, Hopkins M, Kimmel BM, Koberna S, Montie CA. How Cleveland Clinic used TDABC to improve value. Healthc Financ Manage. 2014;68:84-88.

2. Kaplan RS, Anderson SR. Time-driven activity-based costing. Harv Bus Rev. 2004;82:131-138, 150.

3. Kaplan RS, Anderson SR. The innovation of time-driven activitybased costing. Journal of Cost Management. 2007;21.

4. Keehan SP, Sisko AM, Truffer CJ, Poisal JA, Cuckler GA, Madison AJ, Lizonitz JM, Smith SD. National health spending projections through 2020: economic recovery and reform drive faster spending growth. Health Aff (Millwood). 2011;30:1594-1605.

5. Lievens Y, van den Bogaert W, Kesteloot K. Activity-based costing: a practical model for cost calculation in radiotherapy. Int $J$ Radiat Oncol Biol Phys. 2003;57:522-535.

6. McLaughlin N, Burke MA, Setlur NP, Niedzwiecki DR, Kaplan AL, Saigal C, Mahajan A, Martin NA, Kaplan RS. Time-driven activity-based costing: a driver for provider engagement in costing activities and redesign initiatives. Neurosurg Focus. 2014;37:E3.

7. Porter ME. What is value in health care? $N$ Engl J Med. 2010;363:2477-2481.

8. West TD, Balas EA, West DA. Contrasting RCC, RVU, and ABC for managed care decisions. A case study compares three widely used costing methods and finds one superior. Healthc Financ Manage. 1996;50:54-61. 